\title{
Las Conferencias Lowell de Kuhn: Un estudio crítico*
}

\author{
(Kubn's Lowell Lectures: A Critical Study)
}

Juan V. MAYORAL

\begin{abstract}
Recibido: 12.04.2012
Versión final: 14.11.2012

BIBLID [0495-4548 (2013) 28: 78; pp. 459-476]

DOI: $10.1387 /$ theoria. 5680

RESUMEN: Ciertas interpretaciones de la obra de Kuhn subrayan su contribución inconsciente al positivismo lógico, lo que es consecuencia de un conocimiento y una crítica superficiales de dicha corriente por su parte. En este artículo critico dicha tesis a partir de un texto inédito de Kuhn: The Quest for Physical Theory (1951), sus conferencias en el Instituto Lowell de Boston y una primera presentación del punto de vista de The Structure of Scientific Revolutions.

Palabras clave: Kuhn; Pearson; Weber; Koffka; Piaget; conducta; intencionalidad; mundos; orientaciones; convención; vaguedad.

ABSTRACT: Some recent scholarship on Kuhn emphasizes his unconscious contribution to logical positivism-a consequence of Kuhn's superficial knowledge and criticism of that school of thought. In this paper I argue against that thesis on the basis of an unpublished text: The Quest for Physical Theory (1951), his lectures at the Lowell Institute, Boston, and an early version of the point of view of The Structure of Scientific Revolutions.

Keywords: Kuhn; Pearson; Weber; Koffka; Piaget; behavior; intentionality; worlds; orientations; convention; vagueness.
\end{abstract}

\section{Introducción}

De acuerdo con Alexander Bird (2000, 2002, 2005, 2008), el naturalismo epistemológico caracteriza la perspectiva de Thomas Kuhn desde The Structure of Scientific Revolutions hasta la década de los setenta, para después convertirse en un enfoque basado en el a priori, que incluso adquirirá por momentos los tintes de un idealismo más hegeliano que kantiano (cf. Bird 2000, 129-130; 2002, 454-455). La perspectiva kantiana acaba por consolidarse en Kuhn de la mano de Paul Hoyningen-Huene (1993), quien interpreta la obra de Kuhn en términos de un punto de vista fenomenista y neokantiano (cf. Bird 2000, 127-130 y 290-291 n. 44; 2002, 455). ${ }^{1}$ Kuhn se aleja así de su inicial naturalismo, lo que imprime a su filosofía de la ciencia un "giro equivocado", de acuerdo con Bird. Este "error" de Kuhn es más marcado, según Bird, en la medida en

* Deseo agradecer a María Amérigo, Julio C. Armero, Yolanda García, José Luis López de Lizaga, Andrés Pandiella, David Pérez Chico, Douglas Quine, Carlos Solís y dos evaluadores anónimos de la revista THEORIA su colaboración en la preparación y corrección de este artículo.

${ }^{1}$ De acuerdo con Bird (e.g. 2008, 26-27, 36-37), una epistemología naturalizada no excluiría de la investigación empírica ningún aspecto de la cognición humana. La admisión de aspectos a priori propia del neokantismo pone un impedimento a dicho supuesto, ya sean dichos aspectos variables o no. Bird (2008) y Hoyningen-Huene (2008) han explorado la aproximación de ambos enfoques, pero aquí los consideraré perspectivas enfrentadas en ese sentido. 
que supone una reafirmación de algunas tesis del positivismo lógico que permanecían latentes en su pensamiento; en concreto, la idea de que el significado de los términos teóricos depende sólo del contexto teórico, y el rechazo de la idea de verdad como correspondencia (2002, 445 y 452; cf. Preston 2004, 329, quien critica esta conexión, y Newton-Smith 1981, 11-12). Para Bird, es cierto que Kuhn ataca ciertos dogmas del empirismo, como el carácter pre-teórico de la observación y la noción del progreso epistémico optimista de las ciencias (2002, 445 y 460). Pero deja otros sin tocar, como los citados.

Bird explica el "giro equivocado" de Kuhn mediante un argumento ad hominem que recurre al cliché de que Kuhn tenía una escasa formación filosófica. Sabía algo de filosofía, aunque poco y principalmente hasta Kant (2002, 445, 455 y 460). De no haber sido así, Kuhn hubiera percibido que el naturalismo de Structure, un claro ataque al positivismo lógico, se reafirmaba y mejoraba, indica Bird, mediante, e.g., el conexionismo, la teoría causal de la referencia (en la que se evita el problema del significado de los términos teóricos arriba indicado) y el fiabilismo epistemológico. Ello le habría ayudado a ofrecer, como después hizo Howard Sankey (1994, 1997, 2008), una perspectiva sólo semántica de la inconmensurabilidad que admite la continuidad de la referencia y el progreso del conocimiento, y que permite superar el relativismo epistémico e incluso defender el realismo científico. También le hubiese ayudado a mostrar, como Bird ha hecho $(2005,2008)$, que los paradigmas son un gran avance en filosofía de la ciencia, mayor que el descubrimiento de la inconmensurabilidad. Pero Kuhn, dice Bird, pasó por alto estos potenciales apoyos de su crítica; abrazó un punto de vista crítico que era contrario a ese naturalismo (cf. Bird 2000, 140-141; Hoyningen-Huene 1993, 46-47) y se asentó en una perspectiva que sólo acentuaba su inadvertida herencia positivista.

Hay tres aspectos de esta interpretación de Bird que este artículo intentará poner en duda. Primero, que la crítica de Kuhn sea específicamente anti-positivista. Segundo, que Kuhn tenía serias lagunas filosóficas en torno a dicha corriente. Tercero, que hay un positivismo latente en Kuhn, que consiste principalmente en su visión del significado de los términos teóricos. Más tarde veremos argumentos en contra de tales afirmaciones, pero ya podemos apreciar que el testimonio de W. V. Quine en 1955 no respalda al menos las dos primeras ideas. Dice Quine:

Tengo buena opinión de Tom Kuhn y lamento que se marche. La suya es la única mente filosófica en la historia de la ciencia de Harvard. [...] Tom se inclina al positivismo, sin las frecuentes simplificaciones, excesivas y optimistas. Su cautela a ese respecto a menudo le hace evitar las generalizaciones y estar mucho más dispuesto a cuestionar las posiciones filosóficas que a proponerlas. Pero tiene sensibilidad filosófica [philosophical sense] y sus estudios de historia de la ciencia tienen motivaciones epistemológicas. ${ }^{2}$

En este testimonio, Kuhn no parece un crítico anti-positivista superficial, sino alguien que estudia la corriente y que sabe lo que considera valioso en ella y lo que no. Esta otra imagen de Kuhn encaja mejor con lo que veremos después.

\footnotetext{
${ }^{2}$ Carta de W. V. Quine a W. R. Dennes, 4 de oct. de 1955, Dossier de Thomas Kuhn, The Bancroft Library, Universidad de California, Berkeley. Reproducido con permiso de Douglas Quine.
} 
Otra interpretación también defiende la proximidad de Kuhn y el positivismo lógico. Me refiero a los trabajos de G. Reisch (1991), J. Earman (1993), G. Irzik y T. Grünberg (1995) y M. Friedman (2001, 2003). Para Friedman (2003, 29-35), en concreto, Kuhn es un historiador que desarrolla la perspectiva de Emile Meyerson (1908) sobre la dialéctica, a lo largo de la historia, entre la exigencia de racionalidad por parte de la mente humana (lo que en Meyerson consiste en satisfacer un principio de identidad) y la resistencia de la realidad externa a la mente (cf. Solís 1997, 9-13). Según Friedman, Kuhn enriquece así la crítica a Kant ya iniciada por el positivismo lógico y su renovada versión del a priori. Y, en efecto, dado que su a priori sintético dinámico se asemeja al a priori relativizado de $\mathrm{H}$. Reichenbach y que adopta una visión de la relatividad lingüística parecida a la de Carnap (1950) (cf. Irzik y Grünberg 1995), a la que dota de una dimensión histórica, la posición de Friedman resulta razonable (cf. Friedman 1999, 60-64; 2001, 30-31 y 41-43; 2003, 28). No es extraño que Carnap aplaudiera Structure, como afirman Earman, Reisch y Friedman. ${ }^{3}$ Desde este punto de vista, aunque Structure fuese concebida como un ataque al positivismo lógico, no constituía un ataque efectivo, sino, al contrario de lo que pensaba Kuhn, apoyaba algunas de sus ideas más centrales. Estamos de nuevo ante un argumento ad bominem semejante al de Bird: hay un anti-positivismo superficial en Kuhn que acaba por producir efectos contrarios a los deseados por él. Para Friedman (1999, 1-3 y n. 3), quien se apoya en Richard Rorty (1979), Kuhn criticaba una versión ingenua del positivismo lógico como la de A. J. Ayer en Language, Truth and Logic.

Mi argumento en las próximas páginas se basa en un texto inédito, pero central, de la etapa formativa de Kuhn, que él consideraba una versión preliminar de Structure: sus ocho conferencias en el Instituto Lowell de Boston (2-30 de marzo de 1951), tituladas The Quest for Physical Theory: Problems in the Methodology of Scientific Research. ${ }^{4}$ Un estudio en profundidad de dicho texto debería ayudarnos a aclarar la relación de Kuhn con el positivismo lógico. En la sección 2 presentaré brevemente los contenidos de Quest. La sección 3 trata la crítica de Kuhn a la epistemología de la tradición empirista. Si bien esa crítica afecta al positivismo lógico, tiene como objetivo primordial un empirismo que se remonta a la época de Francis Bacon. La sección 4 examina la crítica de Kuhn a la teoría del significado de los términos teóricos del positivismo lógico (crítica que intenta ser coherente con su perspectiva epistemológica). Nuevamente, no observamos un intento de abandono radical del positivismo lógico, sino una matización de su utilidad y una clara cautela con sus abusos. Podremos ver que no se trata de un ataque ingenuo y desinformado. El artículo se cierra con una recapitulación de lo visto.

\footnotetext{
${ }^{3}$ Para una visión escéptica hacia la respuesta de Carnap, cf. Pinto de Oliveira (2007). Kuhn $(2002,270)$ consideraba que la reacción de Carnap había sido "mera cortesía".

${ }^{4}$ Todos los textos de dichas conferencias y otros extractos de TSKP reproducidos a continuación se ofrecen con permiso de los Institute Archives and Special Collections, MIT.
} 


\section{Los contenidos de Quest}

Nuestro estudio de Quest se centrará en sus cuatro últimas conferencias. ${ }^{5}$ La lista completa de todas las sesiones es, no obstante, la siguiente:

I. Introducción: ciencia de libro de texto y ciencia creativa

II. Los fundamentos de la dinámica

III. La prevalencia de los átomos

IV. "El principio de plenitud": fluidos sutiles y campos físicos

V. Evidencia y explicación

VI. Coherencia y visión científica

VII. El papel del formalismo

VIII. Cánones de investigación constructiva

Los contenidos se dividen en dos grupos. Las conferencias II-IV ofrecen material histórico sobre (II) el cambio de enfoque en el tratamiento del problema del movimiento de Aristóteles a Galileo (y, con ello, el cambio de problema en sî), (III) el uso de un programa de explicación atomista de los fenómenos físicos y sus diferencias con (IV) el apoyo en hipótesis físicas basadas en medios continuos, como los fluidos sutiles y los campos. Las tres conferencias de este grupo constituyen un fondo de evidencia histórica sobre el que Kuhn edificará sus argumentos teóricos.

Estos constituyen el contenido de las conferencias restantes. La introducción (I) ofrece una crítica de la idea de la ciencia que surge de la metodología empirista desde Francis Bacon y pasa por John Locke y John Stuart Mill hasta llegar a nuestros días, aunque la presa de los ataques de Kuhn es Karl Pearson y su The Grammar of Science (1900), como lo había sido para su mentor, James B. Conant (1947, 5-15 y 111-115). Para su reconstrucción de la llamada "ciencia creativa", frente a la "ciencia de libro de texto" (1951, I, 5-6), más afín al empirismo de Pearson, Kuhn, dice, empleará las cuatro últimas conferencias, cuya base empírica se encuentra en las tres históricas previas (1951, I, 21).

Las cuatro últimas conferencias se ocupan de la experiencia y el lenguaje científicos. La quinta introduce y describe el concepto clave de "orientación científica" (1951, $\mathrm{V}, 6$ y ss.), esencial en el programa naturalista de Kuhn, así como su patrón de desarrollo discontinuo de la ciencia, basado en la acción de las orientaciones: su "dinámica de las ideas científicas" (1951, V, 18-45), de inspiración fleckiana, que será el núcleo del futuro argumento de Structure. La sexta conferencia se ocupa de la explicación naturalista de las orientaciones y la séptima habla primero de la naturaleza y utilidad de los lenguajes formales en la ciencia creativa, para después iniciar una crítica del abuso de los mismos con fines metodológicos, un mensaje contrario a los programas de reconstrucción racional mediante la lógica, como el de Carnap. Las últimas páginas de la séptima conferencia (1951, VII, 31 ss.) y toda la octava presentan su concepción del lenguaje científico y su estructura semántica. Este conserva rasgos del lenguaje natural, como su vaguedad y el origen intencional del significado. Kuhn muestra una postura opuesta a la perspectiva positivista del significado de los términos teóricos.

${ }^{5}$ Sobre Quest, cf. Andresen (1999, S61-S63) y Marcum (2005, 30-36). 


\section{Orientaciones cientificas y mundos conductuales}

\subsection{Justificación y subjetividad}

Kuhn comienza Quest con la tesis de que la enseñanza de las disciplinas científicas recurre a un argumento basado en la autoridad testimonial de los expertos (1951, I, 12). El libro de texto y el laboratorio son herramientas que sirven de apoyo al testimonio experto (1951, I, 10-11) y que ayudan a forjar la ficción de que los enunciados claves en una teoría, como las leyes científicas, están claramente justificadas. Pero lo cierto, dice Kuhn, es que el aprendiz cede al argumento de la autoridad en cierto punto. Si no lo hiciera, el experto no dispondría de una base justificativa final. Pese a todo (1951, I, 12), dice Kuhn, un estudiante puede tener dudas razonables acerca de la justificación de dicho enunciado, pues en el fondo la consistencia de los enunciados de la teoría en cuestión (tanto interna como externa, i.e., en su relación con otras teorías auxiliares) y la coherencia de las predicciones y las observaciones sólo sustentan la confianza en la fuente de información: los expertos. Esto no es un problema en términos pedagógicos, pero sí en términos epistemológicos. Es un error pensar que en ese entramado de enunciados y recursos materiales encontramos los elementos suficientes para una justificación de las creencias científicas al modo empirista. Puede que esta estructura nos parezca engañosa, pero, dice Kuhn, no hay un procedimiento alternativo para comunicar las creencias científicas que genere la actividad científica por todos conocida, pues este método pedagógico permite examinar individualmente el (y dudar del) apoyo justificativo de ciertos enunciados claves en ciencia e iniciar nuestra investigación a partir de ahí. El problema es del empirismo: haber elaborado su metodología a partir de una (útil) ficción epistemológica con fines pedagógicos. Este es un problema del que, dice Kuhn (1951, I, 21), no será posible redimir al empirismo.

A partir de aquí, Kuhn inicia su propio camino de reforma. Su primer objetivo es el ideal de clasificación desprejuiciada de los hechos (1951, I, 1-5). El ideal, dice Kuhn, ya está presente en Francis Bacon y no sólo llega a Pearson, que es un fiel defensor de la "auto-eliminación" en el juicio (1900, 6; cit. por Kuhn, 1951, I, 1 y 26), sino a la más elaborada filosofía empirista de la ciencia del siglo XX (1951, I, 3). Kuhn sigue a Conant (1947) en ello. El mensaje de Kuhn es claro al respecto incluso ya un par de años antes de Quest. En su cuaderno de notas de la Society of Fellows, en una sección dedicada a The Methodology of the Social Sciences de Max Weber, con cuya teoría del método halló muchos puntos en común, anota Kuhn (21 de junio de 1949, 26; cursivas y corchetes míos):

Nótese que (Weber [1949], 115) la transición a la subjetividad en la evaluación de la física de la que soy partidario es comparable a la transición en historia desde el supuesto [1] de que los historiadores simplemente reúnen hechos que están "ahî" al reconocimiento [2] de la subjetividad inherente a la reunión, ordenación y evaluación de estos hechos.

Kuhn, por lo tanto, busca en el estudio de la ciencia una transición metodológica parecida a la expuesta por Weber, que va de un supuesto dogmáticamente sostenido [1] sobre una recolección de hechos en la que la intencionalidad no cumple papel alguno a la admisión de [2] una selección intencional de los hechos significativos. Asumir esa “subjetividad”, dirá Weber $(1949,82)$, no impide la construcción de un conocimiento 
causal. Kuhn aplica el mismo análisis epistemológico al conocimiento físico, y en ese preciso sentido de Weber: no por ello se asume que el científico se mueve en un halo de irracionalidad. Conviene subrayar este punto en particular. Kuhn lo hace al comienzo mismo de Quest (1951, I, 4-5):

Me han educado como físico, he investigado y estoy comprometido con la creencia en que el conocimiento científico es buen conocimiento, conocimiento útil y, sobre todo, conocimiento acumulativo. Creo que el trabajo de los científicos ha dado como resultado una comprensión cada vez más detallada y de mayor alcance de las operaciones de la naturaleza y que este progreso se puede describir objetivamente de tal modo que un científico del siglo XVII, XVIII o XIX que se enfrentase a un manual con las teorías científicas contemporáneas admitiría sin reticencias que la ciencia ha recorrido un largo trecho desde su propia época.

Para este joven Kuhn, el conocimiento es acumulativo. Kuhn, como Conant, es aún un optimista en términos epistémicos y, para él, hay modos de exponer los logros científicos que convencerían a cualquiera del avance de las ciencias. Kuhn no parece defender la discontinuidad de la referencia y el consiguiente efecto de inconmensurabilidad propio del cambio histórico revisionista. Tampoco hay indicios de una tendencia al relativismo. A decir verdad, esta declaración al comienzo de Quest lo sitúa en el extremo opuesto de L. Fleck $(1979,9-10,21,38-39,62)$ a este respecto. Pero Kuhn destaca en Quest la importancia de atender a la intencionalidad subyacente a nuestra reconstrucción científica de la realidad.

Su indagación de la intencionalidad recurre al principio a términos como "precondición" o "prejuicio", que son extraídos del propio Pearson (Kuhn 1951, I, 25; V, 1). Sin embargo, Kuhn pretende desvincular esa idea, básica en su explicación, de que el científico ya posee nociones preconcebidas sobre la naturaleza de la ciencia en que trabaja y de su objeto de estudio (nociones como que una ciencia es nomotética, por ejemplo, o que sólo hay ciertas propiedades significativas, teóricas u observables, del objeto, etc.) de la carga peyorativa que Pearson y el sentido común asignan a la idea de "prejuicio" (1951, I, 25). Por ese motivo, un término como "orientación científica" es moneda común en Quest. Representa con la mínima carga peyorativa el punto de vista intencional en la ciencia. Veamos más en detalle estas orientaciones.

\subsection{La naturaleza de las orientaciones cientificas}

Empecemos ofreciendo una definición funcional de las orientaciones científicas. Una orientación científica condiciona una parte de la información perceptiva entrante (aquella que tiene que ver con el campo de investigación de un científico) y contribuye al desarrollo, con una intención determinada, de los enunciados básicos de una teoría científica, de las definiciones a las leyes (si las hay), y del instrumental de observación y experimentación usado en ella; presenta problemas y acota el modo en que pueden ser resueltos, de modo que, en cierto modo, adoptar una orientación es asumir (incluso prever) una cierta forma de solución (1951, V, 13-14). Una orientación es, así pues, un precursor cognitivo de una teoría científica (o varias). Tiene un carácter cualitativo y es selectiva y parcial en cuanto a la información relevante.

Hay varios ejemplos de su actividad en las conferencias II-IV. En estos ejemplos queda patente el carácter parcial de una orientación. Como dice Kuhn, hay orientaciones que han sido predominantemente cosmológicas (1951, V, 6-8), pues comprometen 
con una cierta estructura y composición del cosmos. Otras pueden ser metafóricas (1951, V, 9-10), e.g., el atomismo, que permite reagrupar fenómenos aparentemente dispares como manifestaciones de un mismo tipo de interacción entre elementos básicos de la materia. $\mathrm{Y}$ aún otras orientaciones poseen un marcado carácter normativo (1951, V, 10-11), ya que establecen el tipo de compromisos ontológicos y explicaciones que son legítimos, mientras excluyen otros como si fuesen, e.g., meros recursos a "cualidades ocultas"; de nuevo el atomismo es un buen ejemplo.

Las orientaciones de Kuhn se parecen a esas partes de su futura idea de matriz disciplinar que denominará "paradigmas metafísicos" (o "modelos") y "valores" (1996, 184-185; 1977, 297-298). No se corresponden, sin embargo, con la idea de "esquema conceptual", al modo de Conant, pues esta es semejante a la de "teoría" de la representación metodológica empirista del conocimiento científico, cuyas hipótesis o modelos explicativos son suplementos de las teorías y no parte de ellas. El esquema conceptual de Conant sólo usa los conceptos imprescindibles en la interpretación de la experimentación (Conant, 1947, 47; 1948, 1, x). Su naturaleza operacional es clara e intenta despojar al esquema conceptual de las cargas hipotéticas. La proximidad del enfoque de Conant al análisis operacional de Bridgman es marcada y explícita (1947, 48 y 131). Kuhn, en cambio, incluye representaciones cognitivas como parte de la función de una orientación.

\subsection{Mundos conductuales}

La naturaleza cognitiva de la orientación en Quest queda más clara mediante el propio recurso de Kuhn para explicarla. Una orientación, dice en su sexta conferencia (1951, VI, 43-47), es una forma especializada, profesionalizada, de lo que denomina aquí "mundo conductual", o "behavioral world" (1951, VI, 21), al modo de Kurt Koffka $(1935,31)$, uno de los autores de psicología de la Gestalt que parece haber leído (cf. su ficha en TSKP 7-8). Si tuviésemos que encontrar una analogía reciente con la idea de mundo conductual de Kuhn, posiblemente el "mundo nocional" de D. C. Dennett (1987, 152-155) sería un buen candidato. Dennett propone la idea de un modelo ficticio de objetos representados que, como en un modelo semántico, son los referentes de las representaciones del sujeto. Un mundo nocional es, para Dennett $(1987,154)$, "un modelo (pero no necesariamente el modelo real, verdadero) de las propias representaciones internas. No está compuesto por representaciones, sino por representados. Es el mundo 'en el que vivo', no el mundo de las representaciones que hay en $m \hat{\imath}$ '. Como señala W. Bechtel $(1991,101)$, en el mundo nocional "todas las creencias que una persona tiene serían verdaderas y todos los deseos de ella serían razonables". El mundo conductual que Kuhn presenta aquí es similar en ambos rasgos: está compuesto por los objetos representados del sujeto y dichos objetos son los referentes de sus representaciones, de las creencias verdaderas, de los deseos razonables. La principal diferencia entre ambos es el uso que cada uno le da a su concepto. El mundo nocional de Dennett es un artificio heterofenomenológico que se construye para interpretar la idea de comprensión de la conducta sin acudir a las actitudes proposicionales ni a la concepción de un lenguaje del pensamiento. Kuhn, por supuesto, no tiene en mente tal 
proyecto. Él sólo intenta explicar la variabilidad de la percepción de los objetos y situaciones cotidianas de acuerdo con los cambios en las necesidades de la conducta.

La primera noticia de este mundo conductual en Kuhn (aunque aún no denominado de esa manera) se puede encontrar en su lectura de Les notions de mowvement et de vitesse chez. l'enfant de Piaget (1946), que Kuhn comentó extensivamente el 14 de junio de 1949 en su cuaderno de notas (1949, 9-16). Es entonces cuando Kuhn desarrolla una vaga idea de, por un lado, un mundo ajeno a la mente que es un trasfondo causal de estímulos, y, por otro, un mundo representado, compuesto por objetos a los que hemos dado forma, a partir de la fuente causal y mediante conceptos (de nuevo, esto recuerda a Koffka). Lo que percibimos, dice Kuhn ahí, lo es como parte del mundo mental $(1949,13-14)$. Ya es tal la importancia de esta perspectiva para Kuhn en esas fechas que sus primeros planes para un libro de filosofía de la ciencia $(1949,31-32)$ incluían capítulos específicos sobre tal distinción.

Así pues, los mundos conductuales de Kuhn están poblados por todos y sólo aquellos objetos que son relevantes para la conducta. ${ }^{6}$ Dado que la conducta se amolda a un medio externo cambiante, el mundo conductual y sus habitantes cambian a medida que lo hace dicho medio, algo que en el individuo constituye el aprendizaje desde la infancia, aquí explorado en clave piagetiana (cf. Kuhn 1951, VI, 36-41). Así, Kuhn dice (1951, VI, 38-39.):

A medida que el niño crece, estos modos de pensamiento dejan de ser adecuados para tratar con la creciente complejidad de los problemas con que se encuentra. El proceso de pensamiento y el mundo conductual concomitante se ven alterados, y el mundo conductual que obtiene es un producto conjunto de la antigua sociedad en la que ha crecido y de los nuevos problemas que puede tener que afrontar como miembro de esa sociedad. Así, [ese nuevo mundo conductual] no tiene por qué ser, ni normalmente será, exactamente el mismo que el mundo conductual de la generación precedente.

Al igual que para Piaget (e.g. 1977, 183) y para Jerome Bruner, otra de las influencias visibles en estas páginas (1951, VI, 17-19), ${ }^{7}$ para Kuhn la información no sorprende a una mente desprevenida, ya sea esta la del infante o la del científico. El mundo conductual simplifica el complejo flujo de estímulos alimentando con ello la información acerca de los objetos que lo pueblan y constituyendo de ese modo un terreno para la acción que, aunque más pobre de lo que dicho flujo permitiría ver, permite predecir. De hecho, afirma Kuhn, siempre tendemos a ofrecer respuestas a toda pregunta dentro de lo establecido en dicho mundo. Siempre se intenta encajar lo incongruente (1951, VI, 17-20), lo que indica que, como Kuhn afirma en Structure $(1996,193)$ asignamos una misma identidad en nuestro mundo conductual a grupos de estímulos que pueden ser completamente diferentes (cf. Hoyningen-Huene 1993, 42 ss.). Cuando algo así no es posible, el mundo conductual debe cambiar. Hay ocasiones en que ese cambio no genera sólo un enriquecimiento de dicho mundo, sino la pérdida de toda una forma de captar la experiencia que hasta entonces había sido útil. Esto, como en Piaget, es parte del proceso de aprendizaje.

\footnotetext{
${ }^{6}$ Lo que influye en el lenguaje. Kuhn emplea aquí (1951,VI,35) ejemplos de B.L. Whorf (cf. Whorf 1956).

${ }^{7}$ Cf. esp. Bruner y Postman (1949) y Bruner, Postman y Rodrigues (1951). Bruner (1951) ofrece una exposición teórica más completa de los supuestos que subyacen a los experimentos de esos artículos y está entre las lecturas de Kuhn de este autor (cf. su ficha, sin fechar, en TSKP 7-8).
} 


\subsection{Ruptura con la tradición}

Las orientaciones científicas son partes de los mundos conductuales de ciertos individuos dedicados a una profesión científica. Su función es ordenar el flujo de estímulos (i.e., el substrato causal de la percepción) y generar con ello una respuesta cognitiva coherente y completa en forma de objetos que son el referente de nuestros términos. Esto se cumple tanto en el caso de nuestra conducta cotidiana como en el de nuestra principal conducta epistémica, la investigación científica (i.e., ese ámbito donde los mundos conductuales son orientaciones).

Pero si es cierto que los mundos conductuales cambian, no sólo se expanden, y que a veces lo hacen radicalmente, incluso perdiendo piezas en el proceso, entonces la pretensión de que el producto de la investigación científica se evalúa epistémicamente en términos de nuestro contacto con las propiedades observables de nuestras teorías, que suponemos externas a nuestra mente, carece de base. Si lo que queremos es mostrar que hay progreso epistémico en la ciencia, primero hemos de reconsiderar el modo en que decidimos que están justificadas nuestras creencias y el modo en que las inferimos a partir de los hechos, porque la versión empirista falla en su premisa fundamental: su concepción del contacto último con los hechos. Si ese fallo es comprobable (y la historia de la ciencia que ocupa a Kuhn las conferencias II-IV de Quest muestra que lo es), entonces el supuesto de que los expertos que enseñan las teorías de la ciencia son sólo transmisores del conocimiento científico está injustificado. Tales expertos también crean y elaboran sus mundos conductuales (u orientaciones), innovan con ellos, enseñan a ver el mundo en que las propiedades observables relevantes son reconocibles, y, en ciertos momentos críticos, partes del mundo tratado por tales teorías también se pierde con ellos. Esta perspectiva de los mundos conductuales y las orientaciones también impide tratar estas en el sentido del a priori propio de Kant, como Kuhn mismo indica (1951, V, 2-3), puesto que la idea que este defiende aquí de las precondiciones del conocimiento científico ha de incluir que estas puedan cambiar. En la perspectiva menos individualizada de Structure, esta idea será la base de la estructura de las revoluciones científicas, de la que en Quest ya hay una primera presentación, como ya indiqué (cf. sección 2).

En las conferencias V y VI de Quest, por lo tanto, Kuhn ofrece las piezas de una crítica explícita a la base epistemológica del método científico empirista. Esta crítica se apoya en la evidencia histórica, pero sus piezas provienen de una psicología del desarrollo (Piaget), de la personalidad (Bruner), y de la forma (Gestalt), que muestra cómo las expectativas, esquemas, hipótesis o mundos conductuales (los podemos llamar de diversas formas según el autor que sigamos) modifican nuestra percepción del entorno. Incluso, como apunta Kuhn, nuestra actitud emocional afecta a nuestra percepción. (Cf. Kuhn, 1951, VI, 16-21.) Kuhn menciona también a Meyerson como pionero del estudio de las orientaciones. ${ }^{8}$

\footnotetext{
${ }^{8}$ No obstante, la filosofía de Meyerson (1908) no era del todo del agrado de Kuhn, al contrario que su trabajo con casos históricos (cf. Kuhn 2000, 286-287). Friedman (2003, 28-34), sin embargo, respalda una conexión estrecha Meyerson-Kuhn amparándose en una cita de Kuhn $(1977,108)$ que, en realidad, no constituye una confesión en el sentido que requeriría la propuesta de Friedman, a la par que
} 
Con esta perspectiva, Kuhn intenta ofrecer un frente sólido ante la idea pearsoniana de clasificación desprejuiciada de los hechos propia del empirismo. Aunque su mentor, Conant, fuese afín a esta corriente, ${ }^{9} \mathrm{Kuhn}$ coincide con este en que comprender la ciencia es comprender primero cómo los científicos se enfrentan a un determinado problema, es decir, qué los predispone a una descripción del mismo y a una clase particular de soluciones. En Kuhn, esto conlleva un enfoque psicológico que asume un punto de vista intencional.

En realidad, los psicólogos son los compañeros de viaje apropiados para este joven Kuhn. No sólo Bruner y Piaget, sino algunos de los que, por su cuenta, trataron de ofrecer una metodología naturalizada que anticipa la psicología de la investigación del propio Kuhn. Cantril et al. (1949a, 1949b, 1949c) son un buen ejemplo de ello. Inspirados en John Dewey y Arthur Bentley y el enfoque transaccional de Knowing and the Known, Cantril et al. propusieron un punto de vista afín en muchos aspectos al de Kuhn en Quest. Este es un enfoque conocido por Kuhn, no sabemos si ya antes de Quest, pero desde luego enormemente valorado por él. ${ }^{10} \mathrm{Y}$ no hay que olvidar a los psicólogos de la Gestalt. Incluidos como parte de las corrientes que reformaron la epistemología (junto a W. James, el citado Dewey y E. Husserl, por ejemplo), contribuyeron a la quiebra de la teoría de los sense data mediante una inversión de las relaciones entre estos y los objetos físicos que supuestamente permiten constituir en nuestra experiencia perceptiva (cf. Firth 1949, esp. 434). Kuhn consideraba que su propio trabajo, inspirado también en los psicólogos de la Gestalt, pertenecía a esa nueva tradición epistemológica que consideraba que, ante un objeto físico, no retenemos el privilegio de inferir dicho objeto a partir de sus rasgos básicos, sólo lo captamos (i.e., lo hacemos parte de nuestro mundo conductual). Esta es una tradición para la que no hay rasgo básico alguno, sólo objetos captados en función de nuestras expectativas (1951, VI, 12-16).

Con ello, Kuhn consideraba ya en Quest (como más tarde lo haría en Structure, $\ \mathrm{X}$ ) que estaba rompiendo con toda una tradición epistemológica arraigada en el siglo XVII y sumándose a un movimiento de renovación en epistemología. Así, Structure tampoco era un ataque específico al positivismo lógico, como le confesara Kuhn a Ernest Nagel en carta poco antes de su publicación. Dicho libro hubiera sido un texto distinto en tal caso, añadía Kuhn. Tal como le dijo a Nagel,

ignora otros pasajes (Kuhn 1977, 11; 1994, 158-159; y el arriba citado) en que Kuhn muestra su postura ante el idealismo de Meyerson. En una ficha bibliográfica de Meyerson (1908) (TSKP 7-8), Kuhn reproduce una actitud parecida hacia este.

${ }^{9}$ Por ejemplo, Kuhn, a diferencia de Conant, criticaba el análisis operacional (cf. Kuhn 1951, VII, 40-41). Kuhn también manifestó sus discrepancias con respecto al análisis operacional de Bridgman en una carta a E. T. Robinson (8 de marzo de 1965, TSKP 4.14). Según parece, Bridgman (1927) le era familiar a Kuhn ya antes de leerlo (en septiembre de 1943), pues era bien conocido en Harvard, pero le resultaba poco provechoso. Cf. su ficha del libro, fechada, en TSKP 9.

${ }^{10}$ Cf. la carta de H. Shands a Kuhn, 30 de enero de 1963 y respuesta de Kuhn a Shands, 12 de marzo de 1963, ambas en TSKP 4.15; cf. también la ficha bibliográfica de los artículos de Cantril et al. en TSKP 7-8. En esa correspondencia, Kuhn no se muestra demasiado entusiasta, sin embargo, con el texto de Dewey y Bentley. 
Acepto tus dos primeras críticas sin reservas (la especificación del "enemigo" y la posterior explicación [explication] de "paradigma"). Intentaré mejorar ambas cosas en la versión final, aunque sospecho que el resultado no te satisfará por completo. Se necesitaría un libro infinitamente más largo y muy diferente para discutir explícitamente con lo que considero que son los productos modernos de la evolución histórica del baconianismo (y también del cartesianismo, curiosamente). Por ese motivo, he preferido tratar con lo que considero que es la imagen original [root image], y ello me deja en la delicada situación de oponerme a una idea de ciencia que ningún filósofo respetable admitiría sostener. Muchos científicos la sostienen, no obstante, y estoy convencido de que subyace a mucha discusión filosófica altamente refinada. ${ }^{11}$

Así pues, Structure estaba dirigido contra toda la tradición baconiana y cartesiana. Quest nos ofrece más detalles de esa crítica. Cuenta, de hecho, como un ensayo preliminar de la misma.

\section{Lógica e investigación}

\subsection{Sobre lógica y lenguaje científico}

Dejando ya de lado los aspectos epistemológicos de la imagen empirista de la ciencia, Kuhn también se ocupa en Quest de su concepción del lenguaje científico. El intento lógico-positivista de supresión de los términos teóricos sin un claro significado empírico le era familiar desde que leyese Language, Truth and Logic de A. J. Ayer al principio de su estancia en la Society of Fellows y ya entonces no coincidía con la intención de eliminar conceptos que, aunque sin un claro significado empírico, eran no obstante útiles para obtener enunciados confirmados. Por su funcionalidad, tales conceptos eran dignos de confianza y no debían ser suprimidos mientras continuasen siéndolo (Kuhn 1949, 2-6).

En Quest, Kuhn expone una teoría del significado de los términos científicos que encaja bien con la perspectiva epistemológica antes examinada y que está en completa armonía con esa crítica a Ayer. Sin embargo, esta crítica, como veremos en seguida, parece superar lo expuesto por Ayer. Sabemos que en 1951 Kuhn ya ha trabajado otros textos de la tradición positivista, e.g., Carnap (1942), Reichenbach (1938) y la compilación de H. Feigl y W. Sellars, eds. (1949) aparecen en su diario de la Society of Fellows; convendría añadir que conoce los textos de Tarski (1941) y Quine (1940). ${ }^{12}$ Kuhn además interactuó a menudo con el propio Quine en esos años. ${ }^{13}$ Esta biblio-

${ }^{11}$ Carta de Kuhn a Nagel, 22 de junio de 1961, 1. Nagel (carta a Kuhn, 4 de junio de 1961, 1-2) decía que autores como R. Carnap, H. Reichenbach o K. Popper, y sus seguidores, no tendrían por qué oponerse a las tesis de Kuhn sobre el desarrollo científico. En Structure se discute la visión baconiana, añadía, no el reciente positivismo lógico. Ambas cartas están en TSKP 25.53.

${ }^{12}$ Kuhn $(1949,1$ y 9; 2000, 283) y Fleck (1979, viii). Se conservan dos fichas de lectura de Kuhn (TSKP 7-8), con breves comentarios, de dos textos más de Carnap (1928a, 1928b). No aparecen fechadas, pero podrían reflejar las lecturas de Carnap que admite haber hecho tras su graduación en 1943 (Kuhn 2000, 305-306).

${ }^{13}$ Kuhn (s.f., 2) recuerda sus conversaciones con Quine, aunque no añade mucho más. Pero dado que, entre 1945 y 1951, Kuhn dedicó tiempo a la lógica y a la filosofía del lenguaje (cf. Kuhn, "Vita", 1 de octubre de 1955, 1; "Intellectual Autobiography", s.f., ambos en el Dossier de Kuhn, en The Bancroft Library, University of California, Berkeley; cf. también Mayoral 2009) y visto el extracto anterior 
grafía es parte de la formación que hay detrás de los argumentos de Quest que veremos a continuación y que también muestran una comprensión bastante profunda de los argumentos positivistas.

En esa parte de Quest (esp. confs. VII-VIII) hay dos tesis principales que exponer. Primero, su defensa de la lógica como herramienta de investigación apropiada. Segundo, su crítica de los intentos de reducir todo el lenguaje de la ciencia a una forma de cálculo lógico interpretado. Esto amenaza con eliminar dos propiedades básicas del lenguaje científico, aquellas que lo convierten en el vehículo apropiado para investigar la naturaleza: su vaguedad semántica y el venir determinado por la conducta. Él mismo las expone del modo siguiente (1951, VIII, 34-35):

De este modo, los sistemas de significado del lenguaje natural, vagos y determinados por la conducta, son uno de los vehículos más importantes para lo que previamente hemos llamado orientaciones científicas. El área de significado estable es un área de lo que consideramos conocimiento seguro [certain knowledge].

Dado que el empirismo, positivismo incluido, intenta suprimir ambos, Kuhn se enfrenta a esa tradición también en este ámbito. Examinemos esas dos tesis por orden.

\subsection{Convención, verdad y lógica}

Con respecto a los usos de la lógica, Kuhn comienza su séptima conferencia repasando la idea actual de sistema lógico como un cálculo interpretado, separando la validez y la verdad lógica y estableciendo que la necesidad de la verdad matemática es la de la verdad lógica y que esta es una verdad por convención. Para explicar estas ideas, Kuhn aborda un ejemplo matemático obtenido de la geometría y expone, mediante un sistema sencillo de dos postulados y tres grupos de símbolos, los rasgos básicos de un cálculo semejante al que se ocupa de los esquemas básicos de inferencia. Estas páginas ofrecen una introducción, breve y muy básica, a la lógica (1951, VII, 4-12 y 18-26). Pero Kuhn exhibe en ellas algunos compromisos en torno a la lógica.

En primer lugar, defender que las verdades lógicas son verdades por convención desmarca a Kuhn de Quine y lo sitúa en un terreno más próximo al positivista (como ya decía el propio Quine). Kuhn no cita a Carnap ni a H. Poincaré, pero la afirmación sobre la verdad lógica pone a ambos en su punto de mira. Para Kuhn, la lógica ha logrado representar adecuadamente convenciones implícitas en nuestro uso del lenguaje, independientes de las palabras, que sólo dependen del modo en que ordenamos estas para facilitar nuestra comunicación. Kuhn, sin embargo, relativiza este convencionalismo a los usos propios de nuestras raíces lingüísticas, mostrando que es posible que haya una cierta relación entre el indo-europeo y el desarrollo de nuestras matemáticas y nuestra ciencia. No está claro, por lo tanto, que hablar de convención nos libere completamente de nuestras raíces históricas y culturales. La convención de la que se ocupa la lógica tiene que ver, al modo de David Lewis (1969), con resolver problemas de coordinación en la comunicación. La lógica no es más que una ciencia que investiga tales convenciones. Por eso, no es sorprendente, dice Kuhn (1951, VII, 29), que su

de la carta de Quine, es razonable suponer que tales campos y su relación con el positivismo lógico fueran objeto de sus conversaciones con este. 
procedimiento sea circular y que empleemos el mismo lenguaje cuya gramática lógica queremos aislar y comprender objetivamente para llevar a cabo dicha labor, pues la circularidad es inherente a todo pensamiento científico. Así pues, ¿por qué no hablar de convención? Kuhn habla de estas ideas del modo siguiente (1951, VII, 27-28):

Las reglas de la lógica formal o del lenguaje formal son convenciones. Son las reglas del juego que jugamos con otros seres humanos cuando nos comunicamos. En sí mismas, no son más necesarias que cualquier otro conjunto adecuado de reglas, pero sin un conjunto tal no hay comunicación posible, o la hay en muy corta medida. No estamos, entonces, obligados a admitir la necesidad del silogismo, pero una declaración de que no lo haremos es un anuncio de que no acataremos las reglas del juego, de que no jugaremos. Esto es, por lo tanto, anti-social en el sentido más literal y conlleva la pena de otros actos anti-sociales, nos priva del privilegio de aprender ciertas cosas de la experiencia de otros.

Confieso que este punto de vista de que las verdades de la lógica son el producto de la convención lingüística no está de modo alguno libre de dificultad [...]. Me adhiero a él en este momento a pesar de mi incapacidad para resolver todas las dificultades simplemente porque estas no me parecen más serias que las que surgen del intento de arraigar la necesidad lógica en el mundo externo o en categorías innatas de la mente.

En segundo lugar, la utilidad de esta concepción de la lógica, o más bien, de la reducción de partes específicas de ciertos lenguajes a estructuras formales del modo descrito, hace que Kuhn defienda a ultranza la utilidad de esta herramienta. La revolución científica que generó esta comprensión de la lógica en el siglo XIX supuso, dice Kuhn, la aportación de un recurso liberador del pensamiento, ya que permitía distinguir los aspectos formales del mismo de otra serie de compromisos conceptuales. Pero Kuhn es tajante aquí: no debemos olvidar que la formalización es un camino transitorio, que tiene un fin específico, i.e., investigar los compromisos inadvertidos de una teoría y explorar nuevos terrenos dentro de una orientación. La lógica nos libera de las intuiciones y nos permite explorar relaciones internas entre conceptos básicos de una teoría desprovistos de aquellas. Pero este juego tiene un objetivo específico, limitado, para Kuhn. Pretender que la lógica ha de estructurar todo el lenguaje es arbitrario y pernicioso.

\subsection{La vaguedad esencial}

Para sostener esta crítica, Kuhn ofrece una concepción propia del lenguaje científico, lo que nos lleva a la segunda tesis. Kuhn huye de una teoría descriptiva del significado como las de Frege y Russell y de algo parecido a una teoría del racimo como la sugerida poco después por Wittgenstein $(1953, \$ 79)$. Alguien podría pensar que entonces defenderá una teoría causal de la referencia, pero tampoco es así. La aplicación exitosa de un término no depende en Kuhn de un elemento externo, sino interno: del objeto de nuestro mundo conductual que es nombrado al principio de nuestro contacto con el mundo real. Su perspectiva sobre el significado es un poco diferente de las vistas (excepto si la vinculamos con la perspectiva de Dennett). Kuhn se opone a la tesis cartesiana de que la aplicación exitosa de un término a un complejo perceptivo dependa de nuestro conocimiento de ciertas reglas semánticas (1951, VIII, 7-9). Nombramos objetos basándonos en nuestra propia experiencia del mundo (i.e. en lo que sabemos de los objetos que pueblan nuestro mundo conductual) y logramos coherencia con los 
usos de otros individuos a medida que nuestra educación nos saca de nuestro inicial egocentrismo cognitivo (como diría Piaget). Esta perspectiva de Kuhn sobre las reglas semánticas le aleja de los criterios empiristas de significado en dirección a un segundo Wittgenstein.

Examinemos la perspectiva semántica de Kuhn a través del lenguaje científico, que a fin de cuentas es el que le preocupa. Un lenguaje científico no sólo nombra objetos, clases y relaciones de un cierto mundo conductual, sino que intenta hacer más exacto el uso de los términos del lenguaje; i.e., intenta precisar lo que es vago en la propia orientación. Para ello, la línea inicial a seguir sería la marcada por Russell y más tarde desarrollada por Carnap, y consistiría en reducir las referencias a objetos por otras que sólo hagan mención de construcciones lógicas. Pero para Kuhn cada paso dado en esa dirección es un paso dado hacia la arbitrariedad. Esta es necesaria si queremos hacer uniforme el uso del lenguaje y suprimir las ambigüedades. Pero nos aleja de lo que cada uno de nosotros queremos decir al aplicar cada término a un objeto del mundo conductual. Detrás de cada término está la historia cognitiva, intencional, del objeto nombrado en cada mundo conductual. Esta no supone una elaboración esencialista del objeto, porque nuestro principal objetivo es discriminar los objetos, diferenciar los miembros de una clase de los de otra en cada situación en que nos encontramos. Para ello, no siempre nos apoyamos en generalizaciones universales, también dependemos de propiedades que no sabemos si sólo poseen algunos miembros de una clase (o incluso aunque sepamos que en efecto es así) y las usamos para decidir la pertenencia a la misma. Tanto las propiedades que llamaríamos "esenciales" como aquellas otras que sólo sirven como indicadores heurísticos para la acción (en ocasiones hasta el punto de ser determinantes de esta) son constitutivos del significado de los términos que usamos. (Cf. Kuhn 1951, VIII, 13-14.)

En una representación visual de esta idea del significado en Quest (cf. 1951, VIII, 16-18) tendríamos que dibujar una serie de áreas concéntricas, que contendrían atributos que irían de ese grupo reducido de cuya aplicación a todos los miembros de la clase estaríamos completamente seguros, a otras zonas que agruparían aquellos otros atributos que suponemos aplicables al menos a algunos miembros de dicha clase. Ya en el extremo exterior se encontrarían los atributos infrecuentes. Pero Kuhn es tajante a este respecto: el significado del término incluye todas y cada una de esas áreas. Eliminar alguna con el pretexto de la exactitud, por deseable y conveniente que a veces pueda parecer (e incluso ser), es mutilar el significado del término, suprimir una parte de nuestra intención en el uso del mismo. "Porque", dice Kuhn (1951, VIII, 30-31),

los nombres, permítanme repetir, no son simplemente etiquetas artificiales y arbitrarias. Son focos de atención en torno a los cuales cristalizan nuestras expectativas sobre los mundos perceptivos, y el acto de nombrar un complejo perceptivo particular es un acto categórico [positive]. Es un enunciado de creencia sobre la historia futura del complejo nombrado.

Es decir, la aplicación de los nombres, de los términos de propiedad, es sólo un reflejo de nuestra postura intencional acerca del mundo. Esta no sólo incluye lo absolutamente seguro, es decir, aquellas partes del significado de un término sobre las que quizá no haya (casi) discrepancia entre los hablantes de un lenguaje. También incluye nuestra 
aplicación tentativa de nombres, esto es, aquella parte del significado que incluso nosotros (individualmente) consideramos arriesgada.

En esta perspectiva de Kuhn, incluso las más pequeñas variaciones causadas por el mundo de los estímulos quedan registradas en algún o algunos mundos conductuales como viejos objetos con características anómalas, o como nuevos objetos que especifican subclases dentro de otras clases más generales. El lenguaje en Kuhn recibe estas variaciones, las acepta y las emplea, ya sea para continuar con una conducta corriente (e.g., ignorando ciertas propiedades anómalas para introducir un objeto que las posea en una clase común, o empleándolas para identificar, aunque sea de forma insegura, dicho objeto), o para modificar nuestro mundo conductual de modo que incluya una nueva clase, una nueva especie. Esto último es, precisamente, lo que un científico lleva a cabo para resolver un problema mediante un cambio en la orientación. Estas propiedades del lenguaje hacen que el significado no sea una entidad comunicada por reglas, sino un modo de expresar aquellos aspectos estratégicos de nuestra conducta que nos llevan utilizar una determinada palabra para nombrar un objeto. En la teoría del significado de Kuhn, este no se identifica con un grupo de descripciones ni con el objeto externo referido (ni, mucho menos, con una combinación de ambas perspectivas, como el descriptivismo causal). El significado en Quest se identifica con la historia intencional del objeto en nuestro mundo conductual y su estabilidad y variación depende de la suerte de dicho objeto en la conducta futura. No es algo susceptible de fijar; no es algo, en otras palabras, asimilable a reglas. Estas se pueden suprimir.

Más aún, se deben suprimir, lo que Kuhn hará en Structure. Kuhn no estaba, ni podía estar de acuerdo con programas de fijación del significado como el de Russell, Bridgman, Carnap o Reichenbach. Ni siquiera hubiera aceptado, ya entonces, una teoría causal de la referencia, porque, como él indicaba en Quest (1951, VIII, 6), la asignación de un nombre a, e.g., un cierto individuo requiere algún refuerzo descriptivo, ya que el sujeto en cuestión envejece y cambia, a veces notablemente. Los programas de fijación del significado con respecto a las observaciones ya disponibles y las propiedades de las que estamos más seguros suponen un obstáculo a la investigación científica, pues la alteración de un sistema de significado es, como ya he indicado, deseable a veces para resolver problemas. Y también es un obstáculo para el aprendizaje en general, pues si bien mantener fijo el significado puede que nos ayude a justificar el progreso epistémico, también impide que los sistemas semánticos evolucionen y sean mejorados. Si reducimos el significado de un término a sólo ciertas condiciones seguras para su aplicación y lo fijamos en ese punto, impedimos que las variaciones relevantes para la conducta sean asumidas por nuestro sistema de significado y de conocimiento del mundo (1951, VIII, 23-30). Dicho de forma más breve, un programa de fijación del significado introduce una rigidez en este que impide asumir pequeñas novedades en torno a los rasgos identificativos del objeto. Desde el punto de vista intencional de Kuhn, por lo tanto, el mismo recurso empirista que es capaz de explicar el progreso epistémico (e.g., apelando a una reconstrucción racional del lenguaje de las teorías científicas) también impide que se produzca. 


\section{Conclusión}

Entre las interpretaciones de la obra de Kuhn propuestas por Bird y Friedman y la aquí presentada hay una diferencia inicial bastante clara. Para aquellos, Kuhn no está bien informado de los avances en epistemología, semántica, etc., que corren en paralelo a su principal labor intelectual, la de historiador de la ciencia. Sin embargo, tras examinar Quest y la restante evidencia manejada, Kuhn aparece como un estudiante de la filosofía que se gana la vida como historiador y que sabe exactamente qué ideas discurren en paralelo a su trabajo de historia, cuáles de ellas han de ser su principal objeto de crítica y cuáles otras le sirven de apoyo.

En Quest, Kuhn critica la tradición empirista en su conjunto, lo que afecta al positivismo lógico sólo en algunos aspectos de su doctrina. La crítica de Kuhn es, así pues, selectiva, como lo es su búsqueda de apoyos estratégicos en epistemología y semántica. En el primer ámbito, Kuhn abraza las críticas filosóficas de la epistemología tradicional que provienen de la psicología porque son críticas que ofrecen una alternativa a la perspectiva heredada; en el segundo, Kuhn asume una concepción de la lógica que le aproxima al positivismo y le aleja, sin embargo, de la crítica de Quine. Kuhn parece consciente de que esta opción puede ser discutible, pero basta para sus propósitos. Kuhn sí critica el abuso del convencionalismo, argumentando primero por qué se da dicho abuso y ofreciendo una alternativa que se asemeja a las posiciones de Wittgenstein. Así pues, la crítica de Kuhn no revela un anti-positivismo superficial y desinformado, sino un ataque a los dogmas de la epistemología y la semántica heredadas que subyacen a la metodología de la ciencia empirista y la tornan ineficaz para explicar el desarrollo del conocimiento científico.

Como alternativa, Kuhn ofrece una perspectiva intencional que obviamente choca con el positivismo lógico de la época. Kuhn intenta que una perspectiva útil en metodología de las ciencias humanas también se aplique y caracterice a las ciencias naturales. Sin duda este plan queda aquí insuficientemente desarrollado. Por ejemplo, la visión optimista del progreso epistémico y el cambio histórico revisionista que aparecen a la vez en Quest pueden revelarse incompatibles, como más tarde defenderá en Structure. De la misma forma, la idea de orientación y la concepción semántica aquí asociada a ella piden que se explique cómo se produce el solapamiento de mundos conductuales que permite hablar de la orientación científica como visión compartida, de los "periodos clásicos" (i.e., la ciencia normal en los términos fleckianos de Quest), de los "periodos de crisis" y de las revoluciones científicas. En otras palabras, esta "dinámica de las ideas científicas" requiere algo parecido a los futuros paradigmas de Kuhn; o mejor, si cabe, sus estructuras léxicas, más maduras. Kuhn, por lo tanto, no ha dado aún el paso hacia la concepción social de la epistemología que caracterizará su enfoque futuro, y aquí se echa de menos. Pero Kuhn ya intenta aquí algo renovador: se cuestiona cuál es el agente epistémico de la ciencia, qué rasgos le caracterizan y qué recursos lingüísticos, comunicativos, emplea. Aunque en 1951 este sea aún más un proyecto que un programa en curso, Quest nos da muestras de la clase de giro que Kuhn intenta imprimir a la filosofía de la ciencia. Y ya parece que, de tener éxito, constituirá un verdadero cambio. 


\section{REFERENCIAS}

Andresen, J. 1999. Crisis and Kuhn. Isis 90: S43-S67.

Bechtel, W. 1991. Filosofía de la mente. Trad. L. M. Valdés. Madrid: Tecnos.

Bird, A. 2000. Thomas Kubn. Chesham: Acumen.

—. 2002. Kuhn's Wrong Turning. Studies in History and Philosophy of Science 33: 443-463.

—. 2005. Naturalizing Kuhn. Proceedings of the Aristotelian Society 105: 109-127.

- 2008. Incommensurability Naturalized. En Retbinking Scientific Change and Theory Comparison. L. Soler, H. Sankey y P. Hoyningen-Huene, eds. Berlin: Springer: 21-39.

Bridgman, P. W. 1927. The Logic of Modern Physics. Nueva York: Macmillan.

Bruner, J. S. 1951. Personality Dynamics and the Process of Perceiving. En Perception. R. R. Blake y G. V. Ramsey, eds. Nueva York: The Ronald Press: 121-147

—. y Postman, L. 1949. On the Perception of Incongruity: A Paradigm. Journal of Personality 18: 206-223.

-. Postman, L., y Rodrigues, J. 1951. Expectation and the Perception of Color. American Journal of Psychology 64: 216-227.

Cantril, H., Ames, A., Jr., Hastorf, A. H., e Ittelson, W. H. 1949a. Psychology and Scientific Research, I: The Nature of Scientific Inquiry. Science 110: 461-464.

- 1949b. Psychology and Scientific Research, II: Scientific Inquiry and Scientific Method. Science 110: 491-497.

-. 1949c. Psychology and Scientific Research, III: The Transactional View in Psychological Research Science 110: 517-522.

Carnap, R. 1928a. Der logische Aufbau der Welt. Berlín: Weltkreis.

—. 1928b. Scheinprobleme in der philosophie. Berlín: Weltkreis.

—. 1942. Introduction to Semantics. Cambridge, Mass.: Harvard University Press.

—. 1950. Empiricism, Semantics, and Ontology. Revue Internationale de Philosophie 4: 20-40.

Conant, J. B. 1947. On Understanding Science. New Haven, Conn.: Yale University Press

- . 1948. Introduction. En Harvard Case Histories in Experimental Science, vol. 1. J. B Conant y L. K. Nash, eds. Cambridge, Mass.: Harvard University Press: vii-xvi.

Dennett, D. C. 1987. The Intentional Stance. Cambridge, Mass.: The MIT Press.

Earman, J. 1993. Carnap, Kuhn, and the Philosophy of Scientific Methodology. En World Changes. P. Horwich, ed. Cambridge, Mass.: The MIT Press: 9-36.

Feigl, H., y Sellars, W., eds. 1949. Readings in Philosophical Analysis. Nueva York: Appleton-Century-Crofts.

Firth, R. 1949. Sense-data and the Percept Theory, Part I. Mind 58: 434-465

Fleck, L. 1979. Genesis and Development of a Scientific Fact. Trad. F. Bradley y T. J. Trenn. Prefacio de T. S. Kuhn. T. J. Trenn y R. K. Merton, eds. Chicago: The University of Chicago Press (ed. orig. 1935).

Friedman, M. 1999. Reconsidering Logical Positivism. Cambridge: Cambridge University Press.

—. 2001. Dynamics of Reason. Stanford, Cal.: CSLI Publications.

—. 2003. Kuhn and Logical Positivism. En Thomas Kubn. T. Nickles, ed. Cambridge: Cambridge University Press.

Hoyningen-Huene, P. 1993. Reconstructing Scientific Revolutions. Trad. A. T. Levine. Prefacio de T. S. Kuhn. Chicago: The University of Chicago Press.

- 2008. Commentary on Bird's Paper. En Rethinking Scientific Change and Theory Comparison. L. Soler, H. Sankey y P. Hoyningen-Huene, eds. Berlin: Springer: 41-46.

Irzik, G., y Grünberg, T. 1995. Carnap and Kuhn: Arch Enemies or Close Allies? British Journal for the Philosophy of Science 46: 285-307.

Koffka, K. 1935. Principles of Gestalt Psychology. Nueva York: Harcourt, Brace \& World.

Kuhn, T. S. 1949. [Handy Notebook]. 31 de marzo-6 de julio de 1949. TSKP 1.7.

—. 1951. The Quest for Physical Theory. Conferencias Lowell, I-VIII. TSKP 3.11.

- 1977. The Essential Tension. Chicago: The University of Chicago Press.

- 1994. Paradigms of Scientific Revolution. Entrevista con G. Borradori en The American Philosopher. Chicago: The University of Chicago Press.

- 1996. The Structure of Scientific Revolutions, $3^{\mathrm{a}}$ ed. Chicago: The University of Chicago Press $\left(1^{\mathrm{a}} \mathrm{ed} .1962\right.$; $2^{a}$ ed. 1970). 
- 2000. The Road since Structure. J. Conant y J. Haugeland, eds. Chicago: The University of Chicago Press.

V. cast.: El camino desde la estructura. Trad. A. Beltrán y J. Romo. Barcelona: Paidós, 2002.

—. s.f. "Advanced Studies and Research". TSKP 25.53.

Lewis, D. 1969. Convention. Cambridge, Mass.: Harvard University Press.

Marcum, J. A. 2005. Thomas Kubn's Revolution. Londres: Continuum.

Mayoral, J. V. 2009. Intensions, Belief and Science: Kuhn's Early Philosophical Outlook (1940-1945). Studies in History and Philosophy of Science 40: 175-184.

Meyerson, E. 1908. Identité et réalité. París: Alcan.

Newton-Smith, W. H. 1981. The Rationality of Science. Londres: Routledge.

Pearson, K. 1900. The Grammar of Science, $2^{\mathrm{a}}$ ed. Londres: Adam and Charles Black.

Piaget, J. 1946. Les notions de mouvement et de vitesse chez l'enfant. París: Presses Universitaires de France.

—. 1977. El juicio y el razonamiento en el niño. Trad. M. Riani. Buenos Aires: Guadalupe (ed. orig. 1924).

Pinto de Oliveira, J. C. 2007. Carnap, Kuhn and Revisionism: On the Publication of Structure in Encyclopedia. Journal for General Pbilosophy of Science 38: 147-157.

Preston. J. 2004. Bird, Kuhn, and Positivism. Studies in History and Philosopby of Science 35: 327-335.

—. 2008. Reading The Structure of Scientific Revolutions. Londres: Continuum.

Quine, W. V. 1940. Mathematical Logic. Cambridge, Mass.: Harvard University Press.

Reichenbach, H. 1938. Experience and Prediction. Chicago: The University of Chicago Press.

Reisch, G. 1991. Did Kuhn Kill Logical Empiricism? Philosopby of Science 58: 264-277.

Rorty, R. 1979. Philosophy and the Mirror of Nature. Princeton: Princeton University Press.

Sankey, H. 1994. The Incommensurability Thesis. Aldershot: Ashgate.

-. Rationality, Relativism and Incommensurability. Aldershot: Ashgate.

-. Scientific Realism and the Rationality of Science. Aldershot: Ashgate.

Solís, C. 1997. La revolución kantiana de Kuhn. Éndoxa 9: 5-30.

Tarski, A. 1941. Introduction to Logic. Nueva York: Oxford University Press.

TSKP $=$ Thomas S. Kuhn Papers, 1922-1996. MC 240. Institute Archives and Special Collections. Massachusetts Institute of Technology. Cambridge, Massachusetts. (TSKP X.Y = TSKP, caja X,carpeta Y).

Weber, M. 1949. The Methodology of the Social Sciences. E. A. Shils y H. A. Finch, eds. Prefacio de E. A. Shils. Glencoe, Ill.: The Free Press.

Whorf, B. L. 1956. Language, Thought and Reality. J. B. Carroll, ed. Cambridge, Mass.: The MIT Press.

Wittgenstein, L. 2001. Philosophical Investigations, $3^{\text {a }}$ ed. Trad. G. E. M. Anscombe. G. E. M. Anscombe y R. Rhees, eds. Oxford: Blackwell (ed. orig. 1953).

JuAn V. Mayoral es profesor ayudante doctor del área de lógica y filosofía de la ciencia en la Universidad de Zaragoza. Previamente, fue becario postdoctoral en el Departamento de Historia y Filosofía de la Ciencia de la Universidad de Cambridge. Preparó su tesis doctoral sobre el desarrollo intelectual de Thomas Kuhn y ha escrito diversos artículos sobre dicho autor. En la actualidad está finalizando un libro sobre La estructura de las revoluciones científicas.

Dirección: Departamento de Filosofía, Universidad de Zaragoza, c/ Pedro Cerbuna, 12, 50009 Zaragoza, España. E-mail: jmayoral@unizar.es 